\title{
UM ALGORITMO PARA FILTRAGEM DO TRONCO EM NUVEM DE PONTOS LASER TERRESTRE DE ÁRVORES DE PINUS spp.
}

Algorithm for filtering the trunk in terrestrial laser point cloud of Pinus spp. trees

\author{
ANDRÉ LEONARDO BORTOLOTTO BUCK ${ }^{1,2}$ \\ MATHEUS NUNES SILVA ${ }^{2}$ \\ CHRISTEL LINGNAU ${ }^{3}$ \\ ÁLVARO MURIEL LIMA MACHADO ${ }^{4}$ \\ RORAI PEREIRA MARTINS NETO ${ }^{5}$
}

${ }^{1,}$ Programa de Pós-Graduação em Engenharia Florestal - UFPR

${ }^{2}$ SF Florestal LTDA - Sul Florestas

Av: Papa João XXIII, 1555

88505-200 - Ipiranga - Lages - SC, Brasil

${ }^{3}$ Departamento de Ciências Florestais - UFPR

Av: Prefeito Lothário Meissner, 900

80210-170 - Jardim Botânico - Curitiba - PR, Brasil

${ }^{4}$ Departamento de Geomática - UFPR

Rua: Cel Francisco H dos Santos s/n

81531-990 - Jardim das Américas - Curitiba - PR, Brasil

${ }^{5}$ Graduando em Engenharia Florestal - UFPR

andre.buck@sulflorestas.com.br; matheus.silva@sulflorestas.com.br;

lingnau@ufpr.br; alvaroml@ufpr.br; rorai.neto@gmail.com

\section{RESUMO}

A varredura laser terrestre vem sendo testada e apontada como alternativa não destrutiva para mensuração de árvores. Variáveis dendrométricas podem ser obtidas a partir de técnicas de modelagem tridimensional. Porém, é necessária a filtragem dos dados para eliminar pontos que não representam a superfície do tronco. Esse trabalho teve como objetivo apresentar uma proposta de algoritmo, baseada em métodos já consolidados na literatura, para filtragem automática da nuvem de pontos do tronco da árvore. $\mathrm{O}$ estudo foi conduzido a partir de doze árvores 
inseridas em um povoamento inequiâneo de Pinus spp. Três estações laser foram utilizadas para o recobrimento de cada árvore. $\mathrm{O}$ algoritmo proposto, denominado de Filtro Distância Máxima (Filtro $D_{\max }$ ), realiza a filtragem do tronco de forma iterativa e em seções da nuvem de pontos. Para o melhor desempenho da automatização optou-se por um algoritmo parametrizado. O Filtro $D_{\max }$ foi testado experimentalmente e os resultados apontaram que as superfícies dos troncos foram efetivamente filtradas até aproximadamente dois terços da altura total das árvores estudadas. A alta densidade de acículas impediu a representação das superfícies dos troncos no terço superior das árvores. A aplicação do Filtro $\mathrm{D}_{\max }$ não alterou as características geométricas dos troncos proporcionando dados à modelagem tridimensional.

Palavras-chave: Laser Terrestre; Varredura Múltipla; Nuvem de Pontos; Filtragem Automática; Modelo Tridimensional do Tronco.

\begin{abstract}
The terrestrial laser scanner has been tested and pointed as non-destructive alternative for measuring trees. Dendrometric variables can be obtained with threedimensional modeling techniques. However, data filtering is necessary to eliminate points that do not represent the surface to the trunk. This paper aimed at presenting a proposal of an algorithm, based on methods already established in the literature for automatic filtering of point clouds of tree trunk. The study was conducted from twelve trees inserted in a stand of Pinus spp with different ages. Three laser stations were used for surveying each tree. The proposed algorithm, called Maximum Distance Filter $\left(\mathrm{D}_{\max }\right.$ Filter), filters iteratively the trunk by sections of the point cloud. For the best automation performance, we chose a parametric algorithm. The Dmax Filter was experimentally tested and the results showed that the surfaces of the trunks were effectively filtered up to approximately two thirds of the total height of the trees studied. The high density of leaves prevented the surface representation of the trunks in the upper third of the trees. The application of Dmax Filter did not change the geometric characteristics of the tree trunk and provided data to the threedimensional modeling.
\end{abstract}

Keywords: Terrestrial Laser; Multiple Scan; Point Cloud; Automatic Filtering; Three-Dimensional Stem Model.

\title{
1. INTRODUÇÃO
}

A utilização do LASER (Light Amplification by Stimulated Emission of Radiation) tem apresentado grande potencial para o levantamento de variáveis dendrométricas de inventário florestal (LICHTI et al., 2000, 2002; LINGNAU et al. 2007). Dentre os benefícios de se empregar equipamentos laser scanner terrestre para mensuração de árvores, destaca-se a confiabilidade posicional dos dados, a redução de erros cometidos pelo operador do intrumento ou até mesmo de diferentes métodos e dispositivos utilizados na medição (SIMONSE et al. 2003). 
O levantamento de dados utilizando laser scanner terrestre para modelagem da árvore pode ser realizado por meio de uma ou de múltiplas estações. A nuvem de pontos levantada utilizando apenas uma estação laser é denominada como varredura simples, resultando em uma cobertura parcial da árvore. A união das nuvens de pontos de varreduras simples, tomadas em diferentes posições em relação ao objeto de interesse, caracteriza a varredura múltipla, resultando em um recobrimento total da superfície do tronco, galhos e a copa da árvore (THIES e SPIECKER, 2004).

A nuvem de pontos de varredura múltipla pode ser processada para obtenção de informações qualitativas de difícil mensuração, como por exemplo: a geometria ao longo do tronco da árvore, a tortuosidade, a inserção de galhos e os danos presentes no tronco (MAAS et al., 2008). Informações quantitativas também podem ser obtidas de um modelo tridimensional do tronco: diâmetros em diferentes alturas e estudos de volumetria sem empregar métodos destrutivos (BIENERT et al., 2006a). No entanto, os pontos da varredura laser representam a superfície do tronco da árvore, objeto de interesse, assim como pontos de galhos, folhas e demais objetos que se encontravam no campo de visada do equipamento, dados que precisam ser eliminados para aplicação da modelagem tridimensional (LITKEY et al., 2008; LIANG et al., 2008).

Dessa forma, o isolamento da superfície do tronco é uma etapa indispensável à construção de um modelo tridimensional do tronco, tornando o desenvolvimento de métodos de filtragem automáticos, componente fundamental para extração de dados quantitativos de uma floresta. O presente estudo propõe um algoritmo de filtragem da nuvem de pontos de varredura múltipla para subsidiar a modelagem tridimensional do volume do tronco de árvores de Pinus spp.

\section{FILTRAGEM E MODELAGEM DE DADOS LASER TERRESTRE}

A filtragem da nuvem de pontos para o isolamento da superfície do tronco pode ser realizada de forma semiautomática ou automática. No primeiro caso a filtragem é alcançada por meio da eliminação de pontos que visualmente não pertencem à superfície do tronco utilizando ferramentas de edição da nuvem de pontos, todavia, essa abordagem é um método manual, estando sujeito a erros do intérprete. No segundo caso, abordado nesse trabalho, algoritmos implementados via programação computacional eliminam os pontos que não pertencem à superfície que se pretende modelar.

Os métodos automáticos de filtragem em nuvens de pontos de árvores visam eliminar pontos com base em conceitos de limiar de distância entre pontos vizinhos, valor de intensidade ou a densidade de pontos ao longo da superfície do tronco (SIMONSE et al. 2003; ASCHOFF et al., 2004).

Um filtro para o isolamento da árvore em nuvem de pontos foi descrito por Bienert et al. (2006b). O método consiste em gerar um cilindro circular reto de altura e raio pré-definidos a partir de um ponto na base da árvore. A superfície do cilindro estabelece o limiar para que pontos sejam eliminados. 
Litkey et al., (2008), utilizaram o ajuste linear aos pontos da varredura laser de uma árvore para eliminar dados com maior desvio em relação a reta ajustada. Pontos afastados da reta no valor de duas vezes o desvio-padrão da distância dos pontos em relação a reta ajustada foram eliminados resultando na filtragem do tronco da árvore.

No desenvolvimento da modelagem do tronco utilizando dados da varredura laser terrestre, um método denominado "Crescent moon" foi desenvolvido por Király e Brolly (2007), o qual consiste em ajustar círculos em seções transversais ao longo do tronco partindo de três pontos cardeais determinados em relação ao centroide do cluster. O objetivo desse método é a modelagem de circunferências ao longo do tronco. Para eliminar as circunferências consideradas como outliers diâmetros maiores que duas vezes o desvio padrão da série diamétrica ajustada são suprimidos. $\mathrm{O}$ método encontra problemas para realizar o ajuste da superfície do tronco na região da copa das árvores, sendo necessária a realização de estimativas por meio do ajuste de regressão.

Pfeifer et al., (2004), Pfeifer e Winterhalder (2004) e Brolly e Király (2009) propõe o uso de formas geométricas para a filtragem e modelagem dos pontos que pertencem à superfície do tronco. $\mathrm{O}$ procedimento trata da utilização do método de mínimos quadrados para minimizar a discrepância da distância Euclidiana entre os pontos e limites do círculo (Equação1) e do cilindro (Equação 2).

$$
\|\mathrm{c}-\mathrm{p}\|-\mathrm{r}=0
$$

Onde:

$\mathbf{c}=$ coordenadas do centro do círculo $\left(\mathrm{X}_{\mathrm{c}}, \mathrm{Y}_{\mathrm{c}}\right)$;

$\mathbf{p}=$ é um ponto sobre o círculo $\left(\mathrm{X}_{\mathrm{p}}, \mathrm{Y}_{\mathrm{p}}\right)$;

$\mathbf{r}=$ raio.

$$
\|(a-q) \times n\|-r=0
$$

Onde:

$\mathbf{a}=$ coordenadas de um ponto no eixo do cilindro $\left(\mathrm{X}_{\mathrm{a}}, \mathrm{Y}_{\mathrm{a}}, \mathrm{Z}_{\mathrm{a}}\right)$;

$\mathbf{q}=$ coordenadas de um ponto no cilindro $\left(\mathrm{X}_{\mathrm{q}}, \mathrm{Y}_{\mathrm{q}}, \mathrm{Z}_{\mathrm{q}}\right)$;

$\mathbf{n}=\left(X_{n}, Y_{n}, Z_{n}\right)$ direção do eixo do cilindro.

A aplicação do método resultou em clusters de dados que representam a superfície do tronco, suprimindo os pontos encontrados fora dos limites das formas geométricas adotadas.

Algoritmos para a modelagem da área transversal de seções ao longo do tronco foram propostos pelos autores Pereira e Pereira (2009), Király e Brolly (2010) e Silva (2011). O método adotado por Király e Brolly (2010), denominado de Free Form Polygon (FFP), consiste em selecionar pontos localizados a uma distância mínima e determinado azimute do centroide de uma seção da nuvem de pontos em determinada altura da superfície do tronco da árvore. Ao se trabalhar a 
aplicação do método $(F F P)$ para o delineamento da área da seção é possível obter apenas pontos que fazem parte do tronco da árvore realizando, de forma simultânea, a filtragem e a modelagem de dados.

Um modelo tridimensional pode ser obtido utilizando a técnica de triangulação entre pontos (ASCHOFF; THIES; SPIECKER, 2004), porém é necessário que a nuvem de pontos seja pré-processada para a remoção de dados que não fazem parte da superfície de interesse.

\section{OBJETO DE ESTUDO}

O estudo foi conduzido a partir das nuvens de pontos obtidas por varredura múltipla laser terrestre de doze árvores inseridas em um povoamento inequiâneo do gênero Pinus spp. localizado no Campus III da Universidade Federal do Paraná UFPR (Figura 1).

Figura 1 - Características do povoamento inequiâneo. a) Povoamento de Pinus spp;

b) Delimitação da nuvem de pontos laser terrestre de uma árvore.

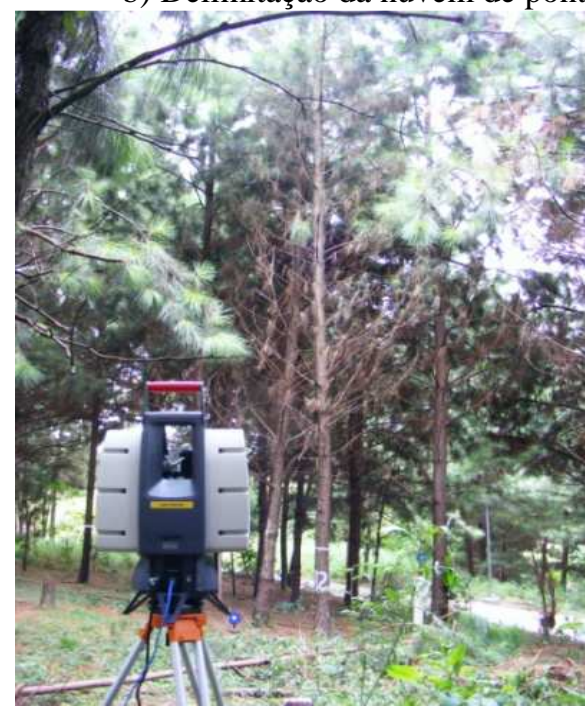

a)

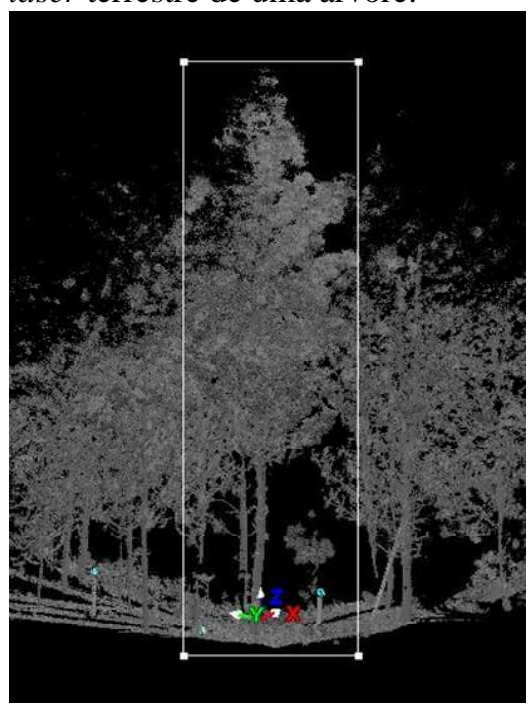

b)

A seleção das árvores submetidas individualmente à varredura laser foi realizada com objetivo de permitir posterior avaliação do comportamento da filtragem da nuvem de pontos ao longo do tronco. Para isso diferentes situações observadas em campo foram contempladas nos indivíduos selecionados: forma irregular do tronco; galhos secos até aproximadamente um terço da altura total da 
árvore; copa composta por alta densidade de acículas; interposição das copas de árvores vizinhas na cena de varredura.

\section{METODOLOGIA}

\subsection{Varredura Laser}

A varredura laser consistiu na aquisição de dados pontuais de toda superfície das árvores, tendo como produto nuvens de pontos de coordenadas tridimensionais (X, Y, Z) das feições presentes no campo de visada do equipamento. Para a coleta de dados foi utilizado o laser scanner terrestre modelo Leica HDS 3000 (Tabela 1).

Tabela 1 - Leica HDS 3000 - características técnicas.

Fonte: adaptado de Leica Scanstation HDS 3000 - User manual (2006).

\begin{tabular}{l|l}
\hline \multicolumn{2}{c}{ Leica HDS 3000 } \\
\hline Taxa de aquisição de pontos & 1800 pontos/segundo \\
\hline Espaçamento mínimo entre pontos & $1,2 \mathrm{~mm}$ \\
\hline Máximo de pontos por coluna & 20000 \\
\hline Máximo de pontos por linha & 5000 \\
\hline Distância mínima de operação & $1 \mathrm{~m}$ \\
\hline Distância máxima de operação & $100 \mathrm{~m}$ \\
\hline Acurácia posicional & $6 \mathrm{~mm} *$ \\
\hline Acurácia distância & $4 \mathrm{~mm} *$ \\
\hline Acurácia angular & 60 micro-radianos \\
\hline * específico para distâncias de até 50 metros.
\end{tabular}

O levantamento de dados foi realizado por meio de três estações de varredura laser terrestre por árvore, totalizando 36 estações e 12 varreduras múltiplas. De acordo com o estudo realizado por Martins Neto, et al. 2013, para o levantamento de dados em árvores individuais a partir da varredura laser, é indicado a instalação do equipamento à uma distância equivalente a altura da árvore, pois em distâncias menores, o ângulo de visada do aparelho é mais inclinado e assim a varredura contempla muitos galhos sobrepostos, os quais acabam por dificultar a aquisição de dados na superfície do tronco nas partes mais altas da árvore. Dessa forma, o equipamento foi posicionado preconizando uma distância equivalente à altura total da árvore mensurada em campo com hipsômetro eletrônico Haglof. A altura das árvores estudadas teve uma amplitude de $12,6 \mathrm{~m}$ à $17,1 \mathrm{~m}$.

Alvos foram posicionados em locais de comum visualização às três estações laser com intuito de apoiar o ajustamento das nuvens de pontos em um sistema de coordenadas local. Os alvos utilizados nessa pesquisa foram do tipo Tilt \& Turn target. $\mathrm{O}$ alvo de superfície quadrada possui lados de $7,62 \mathrm{~cm}$, e o de superfície circular apresenta $15,24 \mathrm{~cm}$ de diâmetro. 
O equipamento laser foi configurado a obter pontos equidistantes de $5 \mathrm{~mm}$ na horizontal e $10 \mathrm{~mm}$ na vertical para a distância em que foi posicionado o equipamento em relação a árvore. Para melhorar a precisão do registro, optou-se pela varredura de maior densidade (espaçamento de $1 \mathrm{~mm}$ ) quando da varredura dos alvos.

\subsection{Pré-Processamento}

O pré-processamento das nuvens de pontos foi realizado sob os dados brutos levantados em campo de acordo com as seguintes etapas: registro; edição dos dados; e translação vertical de coordenadas.

\subsubsection{Registro}

O registro consiste na unificação das nuvens de pontos sob um mesmo sistema de coordenadas local, usualmente idêntico ao da primeira nuvem processada. Essa tarefa é executada por meio do software Cyclone, o mesmo que controla o equipamento laser scanner, e faz uso do centroide de pelo menos três alvos.

\subsubsection{Edição dos Dados}

A nuvem de pontos resultante da varredura múltipla representa a árvore e todos os demais objetos que se encontravam presentes no ângulo das três visadas do equipamento. A árvore de interesse foi identificada e desassociada da nuvem de pontos. Essa etapa foi realizada de forma semiautomatizada, utilizando ferramentas de edição do software Cyclone , como por exemplo, seleção e recorte (Figura 2).

Figura 2 - Isolamento da árvore na nuvem de pontos.

Fonte: BUCK (2012).

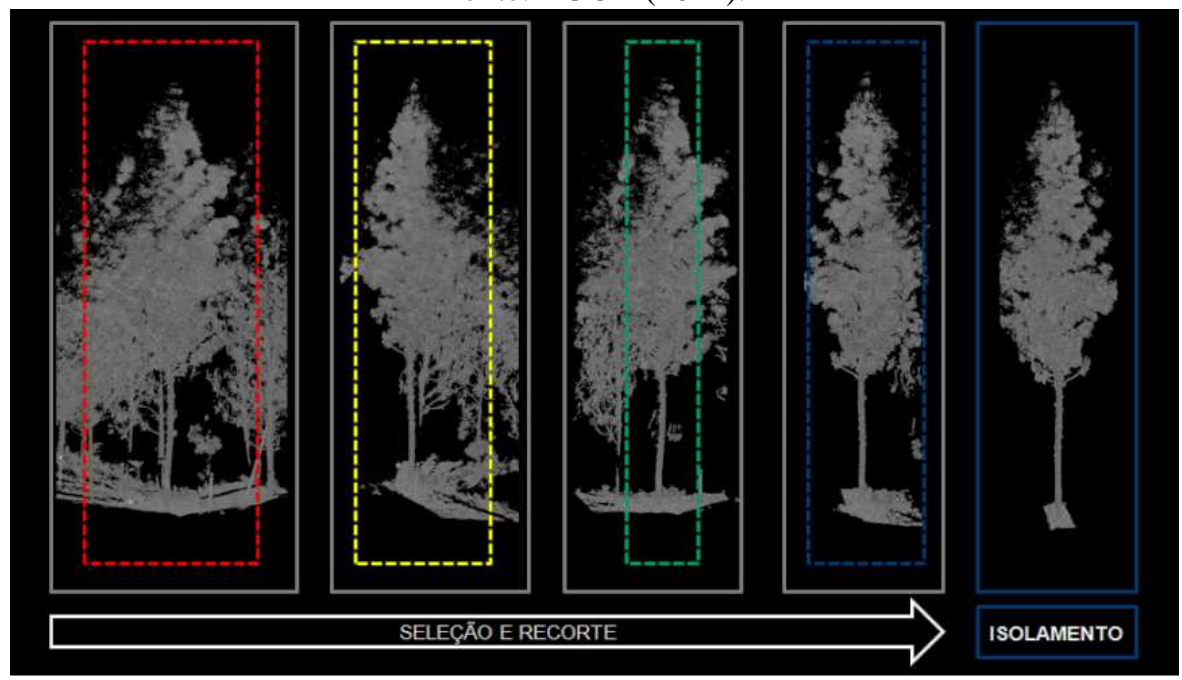

Bol. Ciênc. Geod., sec. Artigos, Curitiba, v. 20, nº 4, p.806-829, out-dez, 2014. 


\subsubsection{Translação vertical de coordenadas}

A nuvem de pontos de varredura múltipla, contêm pontos de coordenadas tridimensionais referenciados por um sistema de coordenada local, relativo ao posicionamento do laser em campo. Devido ao fato de que a altura da árvore é representada pela diferença linear entre o ponto de maior cota, definido pelo ápice da copa, e o ponto de menor cota, localizado na base da árvore junto a superfície do terreno, foi realizada a translação vertical das coordenadas tendo como origem $(\mathrm{Z}=0)$ um ponto localizado na base da árvore.

\section{ALGORITMO: FILTRO DISTÂNCIA MÁXIMA (FILTRO D}

A proposta do algoritmo Filtro Distância Máxima (Filtro $D_{\max }$ ) é a filtragem automática da nuvem de pontos de varredura múltipla para prover dados à modelagem tridimensional do tronco da árvore (BUCK, 2012). O algoritmo está estruturado em etapas compostas por definição de parâmetros, decisões, e instruções iterativas que são executadas no processamento de filtragem dos dados (Figura 3).

Figura 3 - Diagrama do algoritmo Filtro Distância Máxima.

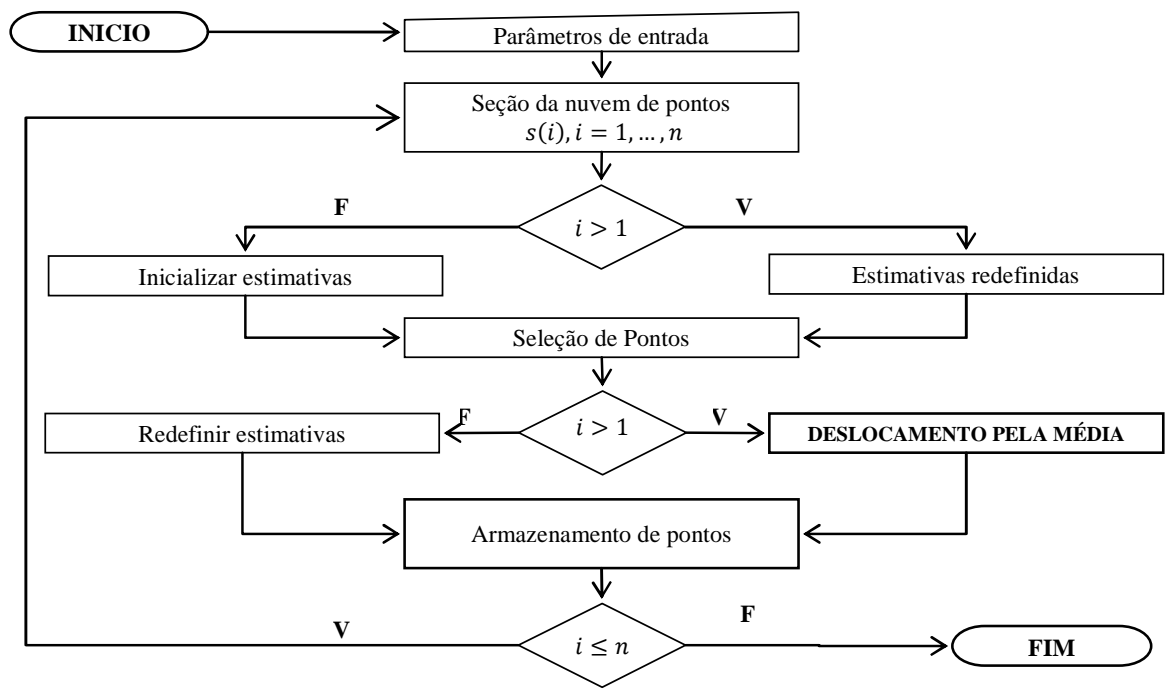

\subsection{Parâmetros de Entrada}

A automatização da filtragem descrita nesse estudo, propõe um algoritmo parametrizado segundo elementos representativos da varredura realizada. Os Parâmetros de entrada estão resumidos na Tabela 2 e explicados com auxílio da Figura 4. 
Tabela 2 - Parâmetros de entrada para o processamento de dados.

\section{PARÂMETROS DE ENTRADA (m)}

\begin{tabular}{l|l}
\hline Espaçamento horizontal & 0,005 \\
\hline Espaçamento vertical & 0,010 \\
\hline Limite da região de busca & 0,500 \\
\hline Espessura da seção & 0,050 \\
\hline
\end{tabular}

Figura 4 - Região de busca: (a) limite da região de busca; (b) região de busca ao longo do tronco, (c) detalhe de uma seção utilizando a região de busca.

Fonte: BUCK (2012)

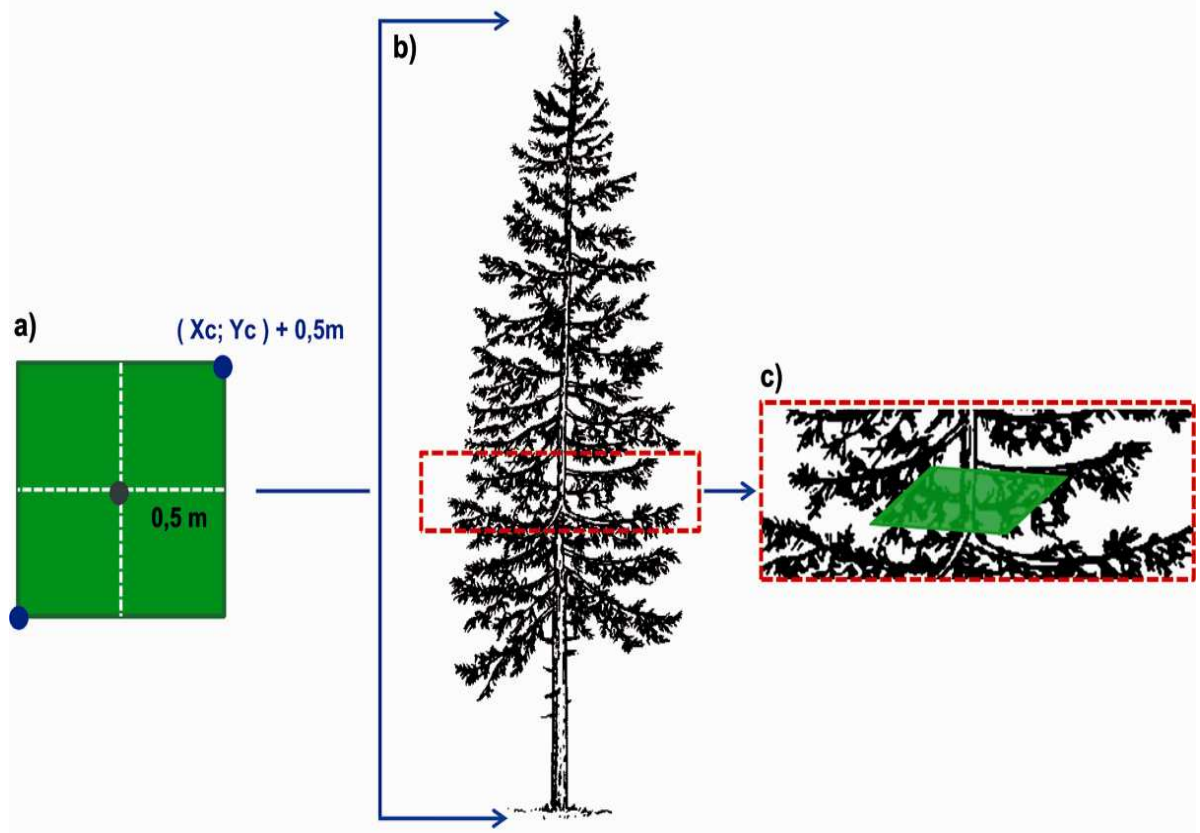

- Espaçamento horizontal e vertical: correspondem às distâncias médias entre pontos adotadas na configuração do equipamento;

- Limite da região de busca: delimita uma região em torno do tronco, definida por um quadrado horizontal;

- Figura 4a): posicionado simetricamente ao redor da árvore;

- Figura 4b): os pontos que se encontram fora desta região de busca são eliminados; 
- Figura 4c): o valor adotado para esse parâmetro teve como objetivo garantir que as seções transversais do tronco se encontrem dentro do limite estabelecido;

- Espessura da seção: representa a variação vertical $(\Delta \mathrm{Z})$ permitida para a formação de um subconjunto de pontos que serão processados agregadamente, compondo uma seção da nuvem de pontos. De certa forma, este parâmetro é dependente da resolução vertical da varredura realizada, e espera-se que o valor definido para este parâmetro seja suficiente para representar adequadamente a seção transversal da árvore.

\subsection{Seção da Nuvem de Pontos}

O processamento de dados da árvore é realizado dividindo o tronco em segmentos verticais. A filtragem é realizada ao longo da superfície do tronco de forma iterativa em subconjuntos de pontos $s(i)$, denominado de Seção da nuvem de pontos (Equação 3),

$$
s(i), i=1, \ldots, n
$$

onde o número total de seções $(n)$ é calculado pela razão entre a altura total da árvore e o valor definido pelo parâmetro "Espessura da seção".

Nesse estudo utilizou-se o valor do ponto de cota máxima no eixo "Z" como a altura total da árvore. Esse procedimento foi aplicado por (LINGNAU et al, $2009 \mathrm{e}$ SILVA, 2011) e caracterizado como uma forma simples e eficiente de se obter a altura total da árvore.

\subsection{Inicializar Estimativas}

A etapa de inicialização de estimativas é realizada para o processamento do primeiro subconjunto de dados $s(i)$ onde $i=1$. Essa etapa consiste em ajustar uma circunferência na primeira seção da nuvem de pontos e obter as seguintes estimativas: coordenadas do centroide $\left(x_{\mathrm{c}}, y_{\mathrm{c}}\right)$ e o raio da circunferência ajustada $\left(r_{\mathrm{c}}\right)$. Dentro de uma seção, a coordenada vertical $(\mathrm{Z})$ do ponto não é levada em consideração.

$\mathrm{O}$ ajuste de uma circunferência à nuvem de pontos, correspondente a determinada seção do tronco da árvore, foi proposto por autores que utilizaram o método de mínimos quadrados (ASCHOFF et al., 2004, LIANG et al., 2008, BIERNET et al., 2006a, BIERNET et al., 2007, LITKEY et al., 2008., SILVA, 2011), buscando assim obter a variável dendrométrica diâmetro.

Segundo SILVA (2011) o "centro ótimo" da circunferência ajustada é aquele em que a variância ou o desvio padrão das distâncias Euclidianas entre esse centro e todos os pontos da seção tem o valor mínimo. $\mathrm{O}$ autor propõe que este enunciado seja resolvido como um problema de pesquisa operacional utilizando a ferramenta 
de análise SOLVER, (Excel e Visual Basic for Applications). A função objetivo (F.O.) é minimizar o desvio padrão das distâncias euclidianas entre todos os pontos da seção e o ponto central estabelecido como referência inicial do centro da circunferência a ser ajustada (Figura 5a).

No processamento da primeira seção da nuvem de pontos as coordenadas iniciais do centroide, necessárias ao ajustamento da circunferência, foram definidas pelo vetor centroide do conjunto de dados (Equação 4),

$$
c^{T}=\left[\begin{array}{ll}
\bar{x} & \bar{y}
\end{array}\right]
$$

onde $c$ é o vetor de médias das coordenadas dos pontos do conjunto de dados em seus respectivos eixos compreendidos na região de busca (Figura 5b). O primeiro ajustamento da circunferência é realizado conforme proposto por Silva, (2011) e resulta em um perímetro que contempla pontos que não fazem parte do tronco (Figura 5c). Para que o perímetro dessa circunferência não ultrapasse o limite definido pelos pontos que delimitam a superfície do tronco, o raio da primeira circunferência ajustada é dividido pela metade conforme descrito em Pfeifer e Winterhalder (2004), (Figura 5d).

Figura 5 - Inicializar estimativas: a) Ajuste da circunferência; b) Determinação do centroide do conjunto de pontos; c) Aplicação do ajuste da circunferência na seção $s(1)$; d) Circunferência com o raio igual a metade de (c).
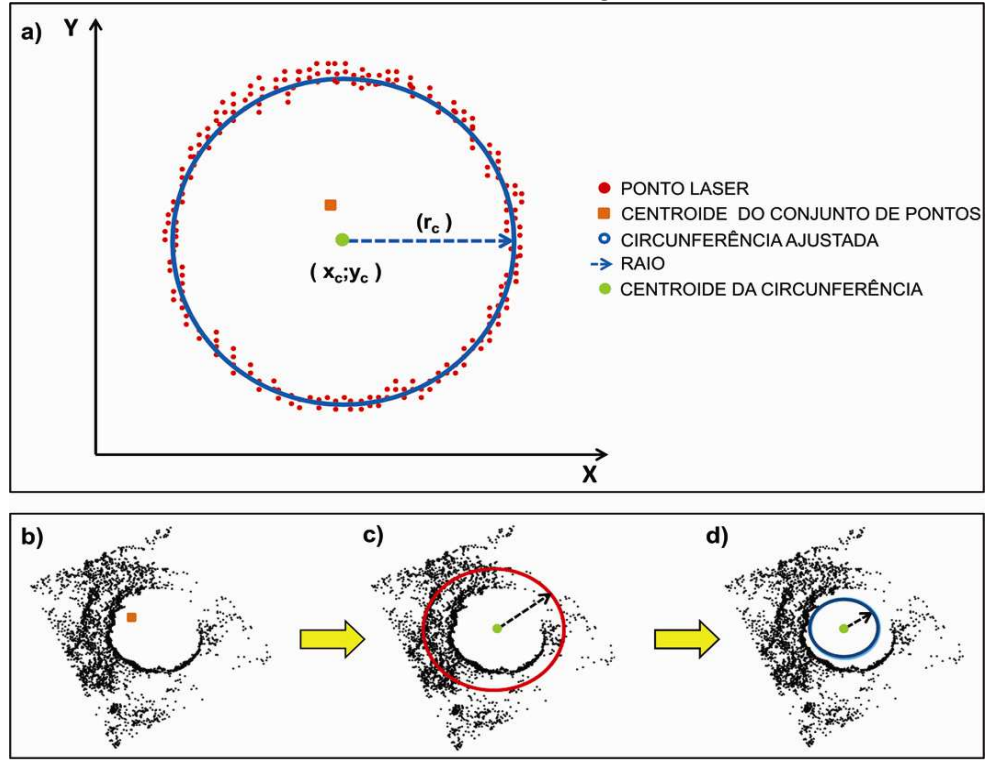

Bol. Ciênc. Geod., sec. Artigos, Curitiba, v. 20, nº 4, p.806-829, out-dez, 2014. 


\subsection{Seleção de Pontos}

O procedimento aqui denominado Seleção de pontos foi aplicado por PEREIRA E PEREIRA (2009), KIRÁLY e BROLLY (2010) e SILVA (2011), o qual busca encontrar a área transversal da seção do tronco da árvore.

A etapa consiste em selecionar os pontos pertencentes ao tronco da árvore utilizando como critério a proximidade entre um ponto de busca (localizado sobre o perímetro da circunferência ajustada) e pontos da superfície do tronco utilizando a distância euclidiana (Equação 5),

$$
\left.d_{\min }=\left[x_{j}-x_{i}\right)^{2}+\left(y_{j}-y_{i}\right)^{2}\right]^{1 / 2}
$$

onde $d_{\min }$ é a menor distância entre um determinado ponto de busca $\left(x_{j} y_{j}\right)$ e um ponto da superfície do tronco $\left(x_{i} y_{i}\right)$ do conjunto de dados da seção (Figura 6).

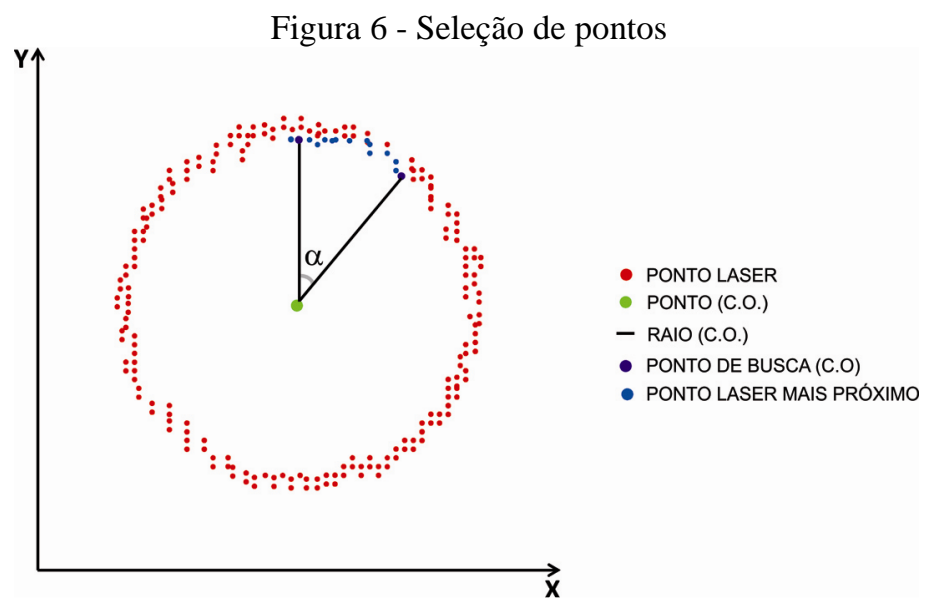

Os pontos de busca são criados aplicando princípios de trigonometria, onde os valores do centro e raio da circunferência ajustada em etapa anterior do algoritmo, são aplicados nas funções trigonométricas em intervalos angulares pré-definidos (de acordo com o perímetro da circunferência ajustada e a resolução horizontal configurada para a varredura) resultando em pares de coordenadas $\left(x_{j}, y_{j}\right)$ que descrevem uma circunferência (Equação 6 e 7),

$$
\begin{aligned}
& x_{j}=x_{c}+r_{c} \cdot \operatorname{sen}(\alpha) \\
& y_{j}=y_{c}+r_{c} \cdot \cos (\alpha)
\end{aligned}
$$

onde: 
$x_{j}=$ coordenada $x$ do ponto $j$

$y_{j}=$ coordenada $y$ do ponto $j$;

$x_{c}=$ coordenada $x$ do centroide;

$y_{c}=$ coordenada $y$ do centroide;

$r_{c}=$ raio da circunferência;

$\propto=$ ângulo entre pontos da circunferência.

\subsection{Redefinir Estimativas}

A etapa Redefinir estimativas utiliza o conjunto de dados obtido na Seleção de pontos que é submetida ao procedimento Ajuste da circunferência, resultando no vetor de Estimativas redefinidas, denotado como $\underline{e}$ (Equação 8).

$$
\underline{e}^{T}=\left[x_{c} y_{c} r_{c}\right]
$$

\subsection{Armazenamento de Pontos}

A etapa consiste na indexação do vetor Estimativas redefinidas (Equação 9),

$$
\underline{e}(i), i=1, \ldots, n
$$

onde $i$ é o número da seção que foi processada e $n$ o número total de seções do tronco submetidas ao processamento de filtragem e no armazenamento das coordenadas dos pontos filtrados pela aplicação do algoritmo nas seções $s(i)$ em uma matriz $F$ de ordem $\sum k_{i}$ x 3 (Equação 10).

$$
F=\left[\begin{array}{c}
F^{(1)} \\
\cdots \\
F^{(i)} \\
\cdots \\
F^{(n)}
\end{array}\right]=\left[\begin{array}{ccc}
x_{11} & y_{11} & z_{11} \\
\vdots & \vdots & \vdots \\
x_{1 k} & y_{1 k} & z_{1 k} \\
\cdots & \cdots & \cdots \\
x_{i 1} & y_{i 1} & z_{i 1} \\
\vdots & \vdots & \vdots \\
x_{i k_{i}} & y_{i k_{i}} & z_{i k_{i}} \\
\cdots & \cdots & \cdots \\
x_{n 1} & y_{n 1} & z_{n 1} \\
\vdots & \vdots & \vdots \\
x_{n k_{n}} & y_{n k_{n}} & z_{n k_{n}}
\end{array}\right]
$$

A matriz $F$ é uma matriz em blocos que armazena os pontos das seções processadas em submatrizes $F^{(i)}$. Dentro de uma submatriz, o primeiro índice indica o número da seção da nuvem de pontos processada e o segundo índice indica a ordem do elemento armazenado na matriz.

Após a conclusão da etapa armazenamento de dados, a continuidade do algoritmo depara-se com a condição de que a seção à ser processada deve ser menor ou igual 
ao número total de sessões à serem filtradas. Caso essa condição seja aceita, procede-se com a segunda etapa do algoritmo, onde será processada a seção que aceita a condição inicial $(i>1)$ como verdadeira, avançando para etapa "Estimativas redefinidas".

\subsection{Estimativas Redefinidas}

O vetor indexado de Estimativas redefinidas $\underline{e}(i)$ é utilizado como referência (BIENERT et al., 2006b) dos valores de centroide e raio da circunferência $\left(x_{\mathrm{c}(i-1)}\right.$; $\left.y_{\mathrm{c}(i-1)} ; r_{\mathrm{c}(i-1)}\right)$, necessários a aplicação da etapa Seleção de pontos quando do processamento das seções $i>1$.

\subsection{Deslocamento pela Média}

O deslocamento pela média consiste em um processo iterativo que realiza a eliminação dos pontos com base nos conceitos Mean Shift Clustering. O método, proposto por Fukunaga \& Hostetler, (1975) utiliza uma função de densidade e probabilidade para encontrar os máximos de um conjunto de dados amostrados delimitados por um kernel. O método consiste em um algoritmo que parte de um ponto $\left(\mathrm{X}_{i}^{0}\right)$ e estabelece uma determinada região (kernel) em torno dele para o cálculo do centro de massa definido como sendo a média aritmética das coordenadas ( $x$ y $)$ dos pontos que estão dentro do kernel (Figura 7).

Figura 7 - Fundamentação do método Mean Shift. Fonte: DERPANIS, 2005 (Modificado).

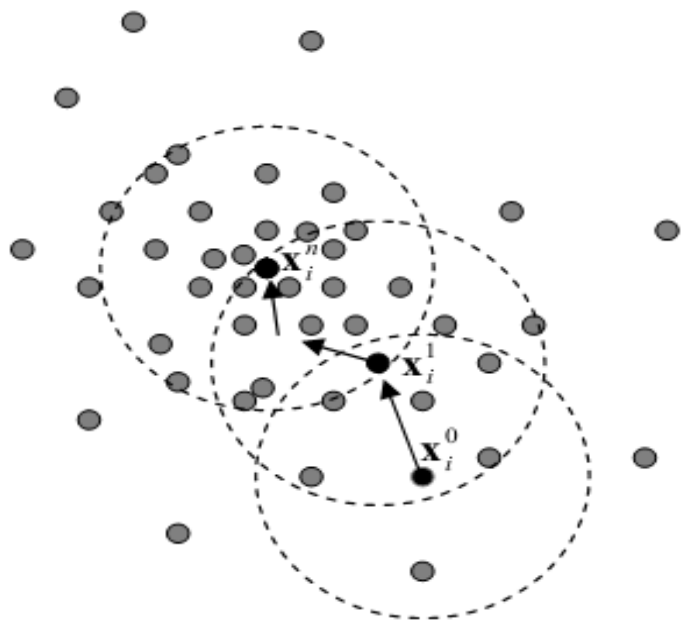

Encontrado o centro de massa $\left(\mathrm{X}_{\mathrm{i}}{ }^{1}\right)$, o algoritmo continua sendo executado e agora realiza o cálculo do novo centro de massa a partir dos dados compreendidos 
dentro da região do novo kernel. As iterações ocorrem até que o centro de massa calculado $\left(\mathrm{X}_{\mathrm{i}}^{\mathrm{n}}\right)$ estabilize e a posição do kernel não seja alterada.

$\mathrm{Na}$ etapa "Deslocamento pela média", inicialmente é realizado o ajuste de uma circunferência ao conjunto de dados advindo da etapa "Seleção de pontos". Pontos localizados abaixo do limiar estabelecido para distância máxima $\left(D_{\max }\right)$, (Equação 11), são filtrados como parte do tronco.

$$
D_{\text {max }}=r_{c}+2 s
$$

onde $r_{c}$ é o raio da circunferência e $s$ o desvio padrão das distâncias euclidianas calculadas entre os pontos da seção e o centroide da circunferência ajustada.

A etapa é realizada dentro de um loop onde o ajuste de uma circunferência e a verificação da condição $D_{\max }$ são acionados em sequência, até que o desvio padrão calculado permaneça inalterado (estabilização do desvio padrão), o que caracteriza a saída do loop e a sequência do algoritmo para a etapa de armazenamento de pontos. A nuvem de pontos filtrada é gerada quando não é possível realizar mais processamentos de seções e o algoritmo é direcionado para o fim.

\section{ANÁLISE EXPERIMENTAL}

A análise experimental foi realizada para avaliar, de forma quali-quantitativa, os resultados da implementação do algoritmo Filtro $\mathrm{D}_{\max }$. Os dados foram avaliados quanto: ao comportamento da filtragem em quatro diferentes seções do tronco, definidas pela base da árvore, $50 \%$ e $75 \%$ da altura total e a ponta; ao número de pontos na nuvem pré e pós-processamento; a altura alcançada pela filtragem em relação à altura total da árvore.

As nuvens de pontos das 12 árvores apresentaram resultados visualmente semelhantes quando submetidas à aplicação do Filtro $\mathrm{D}_{\max }$. Analisando a disposição da nuvem de pontos de medição laser bem como o resultado da aplicação do filtro para uma árvore (Figura 8), é possível observar que o algoritmo filtrou o tronco até aproximadamente dois terços da altura total da árvore.

É importante destacar que as características geométricas da forma do tronco foram mantidas, evidenciando o alto nível de detalhes da varredura laser. A ausência de pontos que representam a superfície do tronco no ápice da árvore, região onde a copa apresenta alta densidade de acículas, apresenta-se como um fator limitante para a filtragem e modelagem do tronco.

Com objetivo de realizar uma análise visual minuciosa ao longo da superfície do tronco foi realizada a divisão do tronco em quatro partes em relação a altura total (25\%) e avaliada cada árvore processada quanto aos critérios: "Pontos dispersos" (D), os quais não foram eliminados na filtragem, e "Partes incompletas" (I), onde o algoritmo pode ter eliminado pontos que representam a superfície do tronco. Uma classificação binária foi realizada atribuindo a pontuação unitária quando observado algum desses critérios na nuvem de pontos filtrada (Tabela 3). 
Figura 8 - Aplicação do Filtro $D_{\max }$ em nuvem de pontos de uma árvore.

Tabela 3 - Classificação segundo a avaliação dos pontos dispersos (D) e dados incompletos (I) visualizados na nuvem de pontos filtrada para cada divisão do tronco da árvore.

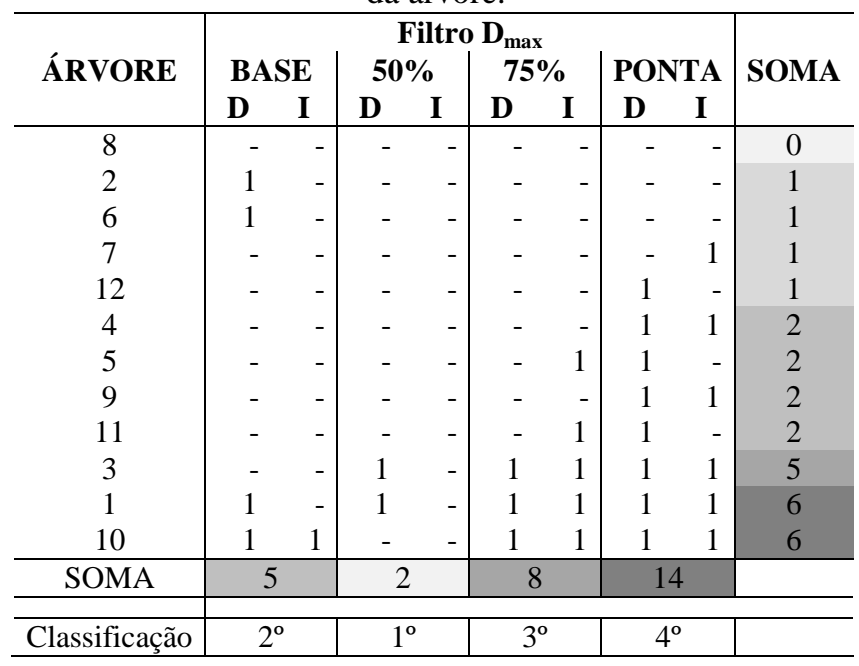


A classificação geral da qualidade da filtragem por árvore (somatória da linha), apresenta a árvore $\mathrm{n}^{\circ} 8$ em primeiro lugar, onde nenhum dos critérios foi observado. As árvores $\mathrm{n}^{\circ} 2$ e 6 apresentaram pontuação unitária para pontos dispersos (D) apenas na divisão BASE, fato que pode ser explicado pela presença de sub-bosque denso no local. As árvores $\mathrm{n}^{\circ} 7,12,4,5,9,11$ apresentaram pontuação unitária nas divisões $75 \%$ e PONTA, esse resultado remete a ausência de pontos que representam a superfície do tronco na copa da árvore. As árvores $\mathrm{n}^{\circ} 3,1$ e 10 apresentaram as maiores pontuações na classificação com maior concentração de pontos dispersos e dados incompletos na base e a partir do início das copas.

As nuvens de pontos originais das árvores $n^{\circ} 3,1$ e 10 foram analisadas e constatou-se que a árvore 1 apresentava em sua base ausência de dados causada pelo desnível do terreno em relação ao posicionamento do equipamento, onde o relevo obstruiu a coleta de pontos do tronco. A árvore 10 apresentava uma deformação (cicatriz) em parte de seu tronco, o que pode ter ocasionando a falta de dados em determinadas seções do tronco. Para a árvore $\mathrm{n}^{\mathbf{0}} 3$, a disposição dos dados originais indica que a varredura laser foi comprometida pela ação do vento, sendo esse intempérie um agravante para utilização de laser terrestre para levantamento da superfície do tronco da árvore. A nuvem de pontos do tronco da árvore forma no espaço tridimensional um eixo vertical perpendicular a superfície do terreno, a presença do vento no momento da varredura pode deslocar esse eixo, resultando em uma nuvem de pontos com conformação irregular (Figura 9)

Figura 9 - Deslocamento do tronco na varredura ocasionado pelo vento.

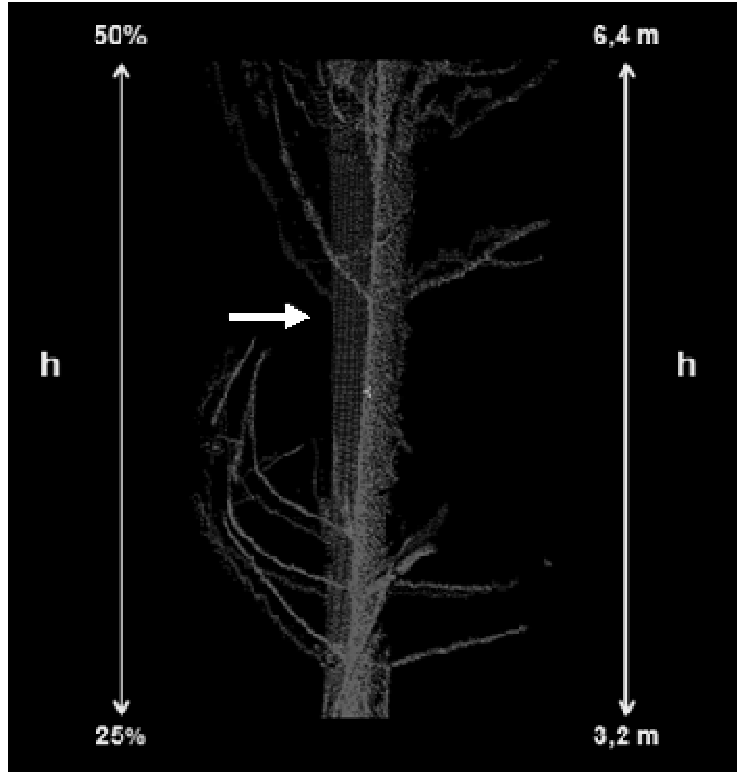

Bol. Ciênc. Geod., sec. Artigos, Curitiba, v. 20, nº 4, p.806-829, out-dez, 2014. 
A classificação geral quanto a qualidade da nuvem de pontos filtrada para a modelagem das seções do tronco (somatória das colunas), apresentou em primeiro lugar a seção correspondente a $50 \%$ da altura total da árvore, superando a seção BASE (segundo lugar), fato que está ligado ao processamento da primeira seção do tronco, onde existe grande quantidade de pontos da superfície do terreno que se confundem com a superfície do tronco. A terceira e quarta posição foram ocupadas, respectivamente, pela divisão $75 \%$ da altura total e PONTA, resultados esperados devido ao início da copa com alta densidade de acículas (Tabela 3).

Os resultados quantitativos do processamento das nuvens de pontos de varredura múltipla das 12 árvores de Pinus spp. que foram submetidas ao processo de filtragem são exibidos na Tabela 4:

Tabela 4 - Resultados quantitativos do processamento da nuvem de pontos da árvore pelo Filtro distância máxima.

\begin{tabular}{c|c|c|c|c}
\hline Árvore & $\mathbf{d}_{(\mathbf{c m})}$ & $\mathbf{h}_{(\mathbf{m})}$ & $\mathbf{N P}_{(\mathbf{n})}$ & ${\text { Filtro } \mathbf{D}_{\max }(\mathbf{n})}$ \\
\hline 1 & 17,7 & 15,5 & 1.772 .735 & 137.824 \\
\hline 2 & 14,4 & 14,7 & 879.389 & 109.656 \\
\hline 3 & 9,9 & 12,8 & 331.974 & 22.024 \\
\hline 4 & 12,1 & 14,4 & 353.994 & 62.167 \\
\hline 5 & 11,6 & 13,8 & 387.169 & 80.698 \\
\hline 6 & 15,5 & 14,1 & 447.419 & 99.856 \\
\hline 7 & 17,9 & 17,1 & 1.191 .101 & 121.620 \\
\hline 8 & 12,5 & 12,6 & 554.105 & 84.147 \\
\hline 9 & 10,2 & 14,2 & 638.400 & 35.937 \\
\hline 10 & 18 & 16,0 & 1.724 .032 & 128.620 \\
\hline 11 & 15,6 & 13,9 & 856.096 & 115.484 \\
\hline 12 & 17,7 & 15,3 & 934.449 & 128.808 \\
\hline \multicolumn{7}{c}{$\mathrm{d}_{(\mathrm{cm})}$ diâmetro da seção transversal medido com suta a 1,30m do solo } \\
$\mathrm{h}_{(\mathrm{m})}=$ altura da árvore obtida na cena de varredura; $\mathrm{NP}_{(\mathrm{n}):}$ número total \\
de pontos na nuvem; Filtro $\mathrm{D}_{\max }(\mathrm{n})=$ número de registros de pontos \\
filtrados.
\end{tabular}

O Filtro $D_{\max }$ foi avaliado quanto a sua capacidade de filtrar pontos que representam o tronco na medida que se depara com a superfície do tronco e galhos, e com a copa densa até a altura total das árvores ( Figura 10).

A altura alcançada pela aplicação do Filtro $D_{\max }$ (Figura10) evidencia que na parte inferior da árvore, a qual é constituída de poucos galhos e acículas secas, não são encontra dos problemas para o isolamento da superfície do tronco. Entretanto, fazendo uma análise da altura relativa alcançada na filtragem foi possível constatar que a superfície do tronco foi efetivamente filtrada até o limite aproximado de $72 \%$ em relação a altura total da árvore, onde ocorre o adensamento de acículas que formam a copa (Figura 11). 
Figura 10 - Altura filtrada pelo método Filtro $D_{\max }$ em relação a altura total, inserção de galhos no tronco e a copa da árvore.

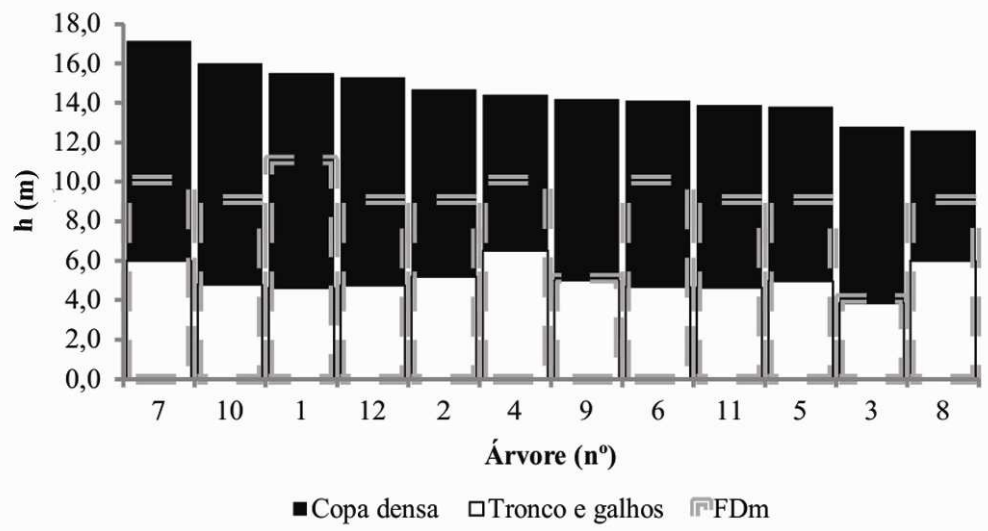

Figura 11 - Percentual do tronco filtrado em relação a altura total das árvores.

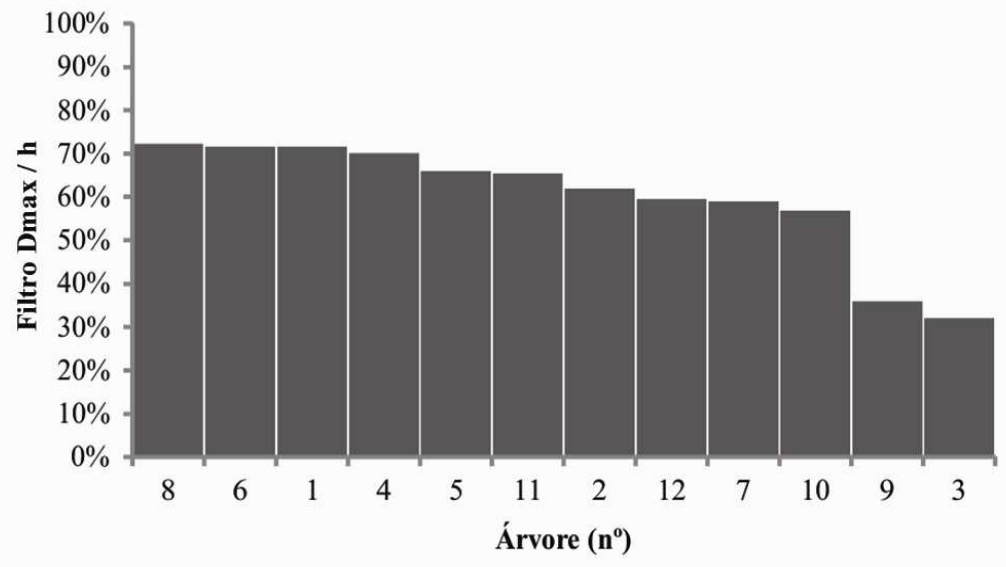

As árvores número três e nove, apresentaram os menores valores percentuais para filtragem do tronco, fato que pode estar ligado com o menor diâmetro do tronco 9,9 e 10,2 cm, respectivamente, quando comparada com as demais árvores, sendo que a árvore três ainda apresenta os problemas já referenciados na análise qualitativa (Tabela 3).

No processamento e análise dos dados foi possível constatar que o Filtro $\mathrm{D}_{\max }$ registra repetidamente as coordenadas dos pontos na etapa do algoritmo denominada como "Seleção de pontos" (Figura 12). 
Figura 12 - Percentual de pontos repetidos em relação aos registros do algoritmo Filtro $D_{\max }$.

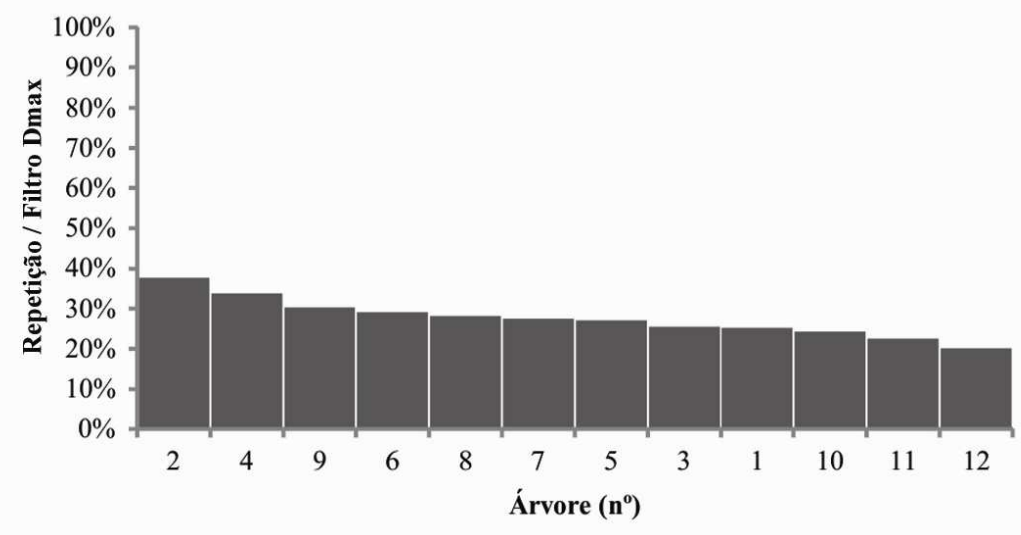

As repetições da seleção de pontos oscilaram entre $20 \%$ e $38 \%$ dos registros na aplicação do Filtro $\mathrm{D}_{\max }$, e tal resultado poderia ser considerado como um problema porém, se habilitada uma restrição de não repetição de pontos, da forma em que foi concebido o algoritmo, poderá ocorrer seleção de pontos que não pertencem a superfície do tronco, comprometendo o resultado final. Dessa forma, o número efetivo de pontos filtrados, desconsiderando o registro de repetições, tornase pequeno quando comparado com a nuvem original de entrada de dados (Figura 13).

Figura 13 - Número de pontos do tronco em relação à nuvem de pontos submetida ao processamento.

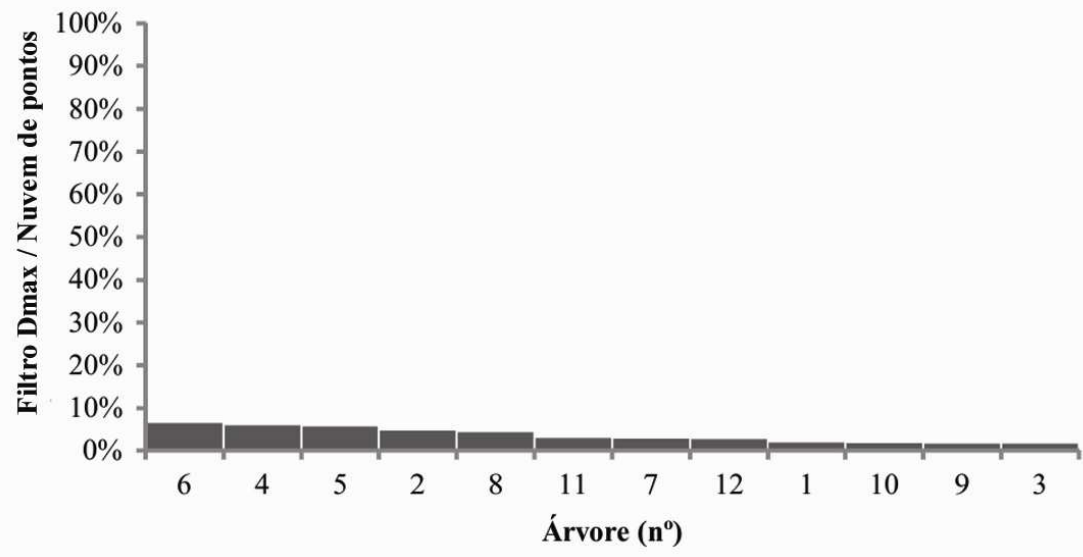

Bol. Ciênc. Geod., sec. Artigos, Curitiba, v. 20, nº 4, p.806-829, out-dez, 2014. 
A aplicação do filtro Filtro $\mathrm{D}_{\max }$ gerou um novo conjunto de dados aptos à modelagem tridimensional para determinação do volume do tronco da árvore. $\mathrm{O}$ conjunto de dados da nuvem de pontos filtrada e o resultado da aplicação da modelagem tridimensional utilizando rede triangular irregular (TIN) para a primeira tora, seção de um metro, são apresentados na Figura 14.

Figura 14 - Nuvem de pontos filtrada e modelo tridimensional (TIN) da primeira tora.

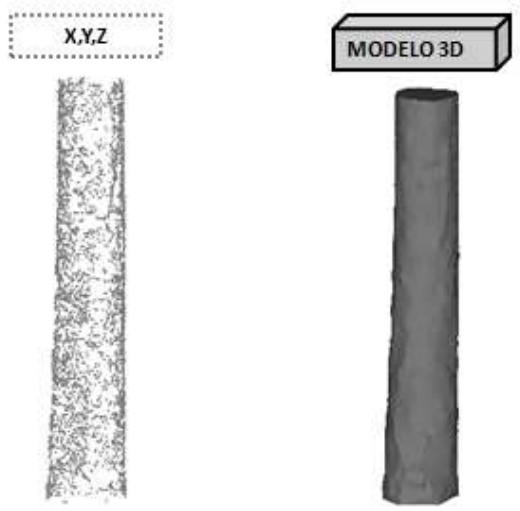

O modelo gerado tem como característica uma superfície suavizada, aspecto que está ligado a redução da densidade de pontos da nuvem original ocasionada pela repetição de registros no processamento Filtro $\mathrm{D}_{\max }$.

O interesse florestal em filtrar todos os galhos e objetos que não pertençam ao tronco se deve ao fato de que para estimativa do estoque das florestas é importante conhecer o afilamento (representado por medidas de diâmetro ou área transversal ao longo do tronco) e a volumetria dos troncos. Neste sentido, é importante observar que um filtro de dados laser terrestre deve proporcionar apenas a superfície do tronco sem ruídos, e que permita a modelagem volumétrica do tronco e a geração de seções transversais a qualquer altura.

O estudo realizou um teste para uma seção de tronco filtrada automaticamente pelo método proposto. $\mathrm{O}$ resultado demonstrou que o método de filtragem gerou dados de qualidade à modelagem tridimensional do tronco.

\section{CONCLUSÕES}

A aplicação do algoritmo Filtro Distância máxima para o isolamento do tronco de árvores do gênero Pinus spp. resultou em dados adequados à modelagem tridimensional onde a nuvem de pontos manteve as características geométricas do tronco da árvore.

O limite encontrado para utilização da filtragem proposta, relativo a variável altura total, foi de aproximadamente $72 \%$, coincidindo com o início da copa densa e 
consequentemente é observada uma ausência de pontos que representam o tronco nas partes superiores da árvore. A alta densidade de acículas, que impede a chegada do pulso laser até o tronco, é apontada como a razão desse problema.

Pode-se concluir que a rotina de filtragem é capaz de isolar o tronco de forma automática e fornecer dados aptos à modelagem, porém, a ausência de pontos que representam a superfície do tronco inviabiliza a sua total reconstituição. Nesse caso é recomendada a aplicação de métodos estimativos do volume do tronco após o adensamento da copa.

\section{AGRADECIMENTOS}

Os autores agradecem ao Programa de Pós-Graduação em Engenharia Florestal da Universidade Federal do Paraná (PPGEF/UFPR), pela infraestrutura disponibilizada para a realização deste trabalho; Ao Departamento de Geologia da UFPR, pela disponibilização do equipamento Laser HDS3000 para a coleta de dados; Ao Programa de Reestruturação das Universidades Federais (REUNI) pelo fomento da pesquisa.

\section{REFERÊNCIAS BIBLIOGRÁFICAS}

ASCHOFF, T.; THIES, M.; SPIECKER, H. Describing forest stands using terrestrial laser-scanning. International Archives of Photogrammetry, Remote Sensing and Spatial Information Sciences v. 35, comm. 5, p. 237- 241, 2004.

ASCHOFF, T.; SPIECKER, H. Algorithms for the automatic detection of trees in laser scanner data. International Archives of Photogrammetry, Remote Sensing and Spatial Information Sciences, v. xxxvi - 8/w2. Freiburg, 2004.

BIENERT, A.; MAAS, H.G.; SCHELLER, S. Analysis of the information content of terrestrial laserscanner point clouds for the automatic determination of forest inventory parameters. In: Workshop on 3D Remote Sensing in Forest, 14-15. Vienna, 2006a.

BIENERT, A., SCHELLER, S., KEANE, E., MULLOOLY, G. AND MOHAN, F. Application of terrestrial laser scanners for the determination of forest inventory parameters. International Archives of Photogrammetry, Remote Sensing and Spatial Information Sciences, 36. $2006 \mathrm{~b}$.

BIENERT, A., et al. Tree detection and diameter estimations by analysis of Forest terrestrial lasescanner point clouds. ISPRS Workshop on Laser Scanning 2007 and SilviLaser 2007. Finland, 2007.

BROLLY, G., KIRÁLY G. Algorithms for stem mapping by means of Terrestrial Laser Scanning. Acta Sylvatica et Lignaria Hungarica, v5, p. 119-130, 2009.

BUCK, A. L. B. Análise de métodos de filtragemem dados laser scanner terrestre aplicados à modelagem tridimensional do tronco de Pinus spp. 2012. 130f. Dissertação (Mestrado em Engenharia Florestal) - Universidade Federal do Paraná, UFPR, Curitiba, 2012.

DERPANIS, K.G. Mean Shift Clustering. 2005. Disponível em: www.cse.yorku.ca/ kosta/.../mean_shift.pdf. Acesso em:25/10/2011. 
FUKUNAGA, K,; HOSTETLER,L. The estimation of the gradient of a density function, with applications in pattern recognition. IEEE Transactions On Information Theory, Madison - N.J., v. 21, n. 1, p.32-40, jan. 1975.

KIRÁLY, G.; BROLLY, G. Tree height estimation methods for terrestrial laser scanning in a forest reserve. IAPRS, v. 36, p. 211-215, 2007.

KIRÁLY, G; BROLLY, G.. Volume calculations of single trees based on terrestrial laser scanning. In: SILVILASER, 10², 2010, Freiburg. Proceedings... Freiburg, 2010. 1 CD-ROM.

LIANG, X. et al. Plot-level trunk detection and reconstruction using one scan mode terrestrial laser scanning data. 2008 International workshop on Earth Observation and Remote Sensing Applications, IEEE, China, Beijing, 30 June2 Jul, 2008.

LICHTI, D. D et al. Benchmark tests on a three-dimensional laser scanning system. Geomatica Res. Aust, v. 72,p. 1-23, 2000.

LICHTI, D. D et al. Ground-based laser scanners: operation, systems applications. Geomatica, v. 56, p. 21-33, 2002.

LINGNAU, C.; NAKAJIMA, N. Y.; DAMAS, B.; SANTOS, D. S. DOS; VINHAL, L. A. Obtenção de parâmetros florestais através de laser terrestre - Novas perspectivas. In: SIMPÓSIO BRASILEIRO DE SENSORIAMENTO REMOTO, 13., 2007, Florianópolis. Anais... Florianópolis: INPE, 2007.

LINGNAU, C.; MACHADO, A.M.L.; BAVARESCO JUNIOR, A.; COLLA, F.L. Determinação de diâmetros a diferentes alturas de árvores individuais a partir de dados laser terrestre. In: Simpósio Brasileiro de Sensoriamento Remoto, 14, 2009, Natal. Anais... Natal: INPE, 2009.

LITKEY, P. et al. Single-scan TLS methods for Forest parameter retrieval. In: Silvilaser 2008, 2008, Edinburgh UK. p. 295 - 304.

MARTINS NETO, R. P.; BUCK, A. L. B.; SILVA, M. N.; Lingnau, C.; MACHADO, A. M. L.; PESCK, V. A. Avaliação da varredura laser terrestre em diferentes distâncias da árvore para mensurar variáveis dendrométricas. Boletim de Ciências Geodésicas, v. 19, p. 420-433, 2013.

MAAS, H.-G.; BIENERT, A.,SCHELLER, S.; KEANE, E. Automatic forest inventory parameter determination from terrestrial laser scanner data. International journal of remote sensing, v. 29, n. 5, p. 1579-1593, 2008.

PEREIRA, A. R.; PEREIRA, R. G. Extração automática de árvores e áreas de secções transversais a partir de dados laser terrestre. Trabalho de Conclusão de Curso(Graduação em Engenharia Cartográfica). Departamento de Engenharia Cartográfica, Universidade Federal do Paraná, 2009.

PFEIFER, N.; GORTE, B.; WINTERHALDER, D. Automatic reconstruction of single trees from terrestrial laser scanner data. International Archives of Photogrammetry, Remote Sensing and Spatial Information Sciences (ISPRS), v. 35, n. 5, part b, p. $114-119,2004$.

PFEIFER, N.; WINTERHALDER, D.. Modelling of tree cross sections from terrestrial laser scanning data with free-form curves. International Archives of

Bol. Ciênc. Geod., sec. Artigos, Curitiba, v. 20, nº 4, p.806-829, out-dez, 2014. 
Photogrammetry, Remote Sensing and Spatial Information Sciences, v. 36, n. 8/w2. Freiburg, 2004.

SILVA, M.N. Aplicação de laser scanner terrestre para determinação de variáveis dendrométricas em Pinus spp. 2011. 126 f. Dissertação (Mestrado) - Curso de Engenharia Florestal, Departamento de Ciências Florestais, UFPR, Curitiba, 2011.

SIMONSE, M., ASCHOFF, T., SPIECKER, H., THIES, M., 2003: Automatic Determination of Forest Inventory Parameters Using Terrestrial Laserscanning. Proceedings of the ScandLaser Scientific Workshop on Airborne Laser Scanning of Forests, Umeå/Sweden, 2003. p. 251- 257.

THIES, M.; SPIECKER, H. Evaluation and future prospects of terrestrial laser scanning for standardized forest inventories. International Archives of Photogrammetry, Remote Sensing and Spatial Information Sciences, v. xxxvi 8/w2. Freiburg, 2004.

(Recebido em janeiro de 2014. Aceito em agosto de 2014). 